\title{
Direct Transfer Patterning of Electrically Small Antennas onto Three-Dimensionally Contoured Substrates
}

\author{
Carl Pfeiffer, Xin Xu, Stephen R. Forrest,* and Anthony Grbic*
}

The rapid expansion of the mobile electronics industry has created a glaring need for devices that are both small and able to communicate wirelessly. The largest component on wireless devices today is typically the antenna. This fact alone has driven numerous antenna miniaturization studies and research efforts into the design and fabrication of electrically small antennas (ESAs): antennas small compared to the operating wavelength. ${ }^{[1-11]}$ An antenna is considered to be electrically small when $k a<0.5$, where $k=2 \pi / \lambda$ is the free space wavenumber, and $a$ is the minimum radius of a sphere that circumscribes the antenna.

A typical ESA consists of a small dipole antenna with an external matching circuit. However, this antenna generally exhibits a narrow bandwidth and relatively low efficiency. ${ }^{[4]}$ Other miniaturized antenna designs include resonant, magnetically-coupled antenna elements packed into a small volume, ${ }^{[8]}$ space-filling curve antennas, and fractal curve antennas. ${ }^{[2]}$ ESAs can be categorized as either volumetric or planar. Volumetric ESAs offer excellent performance, whereas planar designs exhibit limited performance: relatively low bandwidths and low radiation efficiencies. ${ }^{[4]}$ Planar designs, on the other hand, are popular since their fabrication is simple and inexpensive. Here, we combine the advantages of these two technologies by developing a simple method to fabricate high performance, volumetric, printed ESAs.

The most common method to fabricate volumetric antennas is to manually bend wires into a desired shape. ${ }^{[3,4,12]}$ However, this fabrication process is inaccurate, potentially expensive, and slow. More recently, a method was introduced to print conductive ink onto a volumetric surface. ${ }^{[1]}$ In that case, the ink used had a conductivity approximately $30 \%$ that of copper, which ultimately limited the antenna efficiency. In addition, since it was a direct write process, every trace was individually drawn, making the process relatively slow. Another method that is used to

C. Pfeiffer, Prof. A. Grbic

Department of Electrical Engineering and Computer Science University of Michigan

Ann Arbor, MI, 48109-2122 USA

E-mail: agrbic@umich.edu

Dr. X. Xu

Universal Display Corp

375 Philips Boulevard, Ewing

New Jersey, 08618, USA

Prof. S. R. Forrest

Departments of Electrical Engineering and Computer Science

Material Science and Engineering, and Physics

University of Michigan, Ann Arbor, MI, 48109-2122 USA

E-mail: stevefor@umich.edu

DOI: 10.1002/adma.201104290 fabricate volumetric antennas is to first print the antenna onto a flexible printed circuit board using conventional photolithography, and then deform the circuit board into a desired shape. ${ }^{[13]}$ However, this method is limited to fabricating relatively flat shapes, since a substantial deformation of the substrate can cause metallic lines to crack. Here, we present a direct transfer patterning process that allows printing by direct stamping of arbitrary patterns onto a contoured substrate. This process can be used to accurately and rapidly fabricate volumetric ESAs on a variety of shaped substrates. In addition to the fabrication of electrically small antennas, this process can also be applied to other wireless technologies such as high gain antennas, antenna arrays, radio-frequency identification devices (RFIDs), metamaterials and transformation optics. ${ }^{[14-16]}$ To demonstrate the value of this process, we designed, fabricated and measured ESAs of various electrically small sizes that operate over several frequency ranges. We show that the antennas fabricated using this direct transfer patterning process have significantly higher performance and are potentially easier to mass produce than previously reported methods of fabricating electrically small antennas on non-planar surfaces.

One important metric for ESAs is their radiation quality factor (Q), which is equal to the ratio of stored-to-radiated energy, and is inversely proportional to the bandwidth. ${ }^{[17]}$ The goal of most ESA research has been to increase efficiency and reduce antenna $Q$ as much as possible. However, there exists a fundamental, physical restriction on the minimum achievable $Q$ for single resonant antennas, viz.:

$Q_{l b}=\eta_{e f f}\left(\frac{1}{(k a)^{3}}+\frac{1}{k a}\right)$

where $2 a$ is the maximum dimension, $\eta_{\text {eff }}$ is the radiation efficiency, and the subscript $l b$ denotes lower bound. ${ }^{[5,7,9,11]} \mathrm{A}$ common metric used to analyze the performance of an ESA is the ratio: $Q / Q_{l b}$. To date, there are several examples of antennas that closely approach the fundamental limit. ${ }^{[1,3,4,8,10,12]}$ We chose to fabricate the spherical helix antenna because it closely approaches this limit, and its design is relatively simple. ${ }^{[3]}$ The spherical helix antenna inductively loads a dipole antenna by winding metallic arms around the surface of a hemisphere. By varying the number of arms and the number of turns each arm makes around the sphere, it can be impedance-matched to $50 \Omega$.

Direct transfer patterning has been previously used to fabricate photodetector arrays on a hemispherical surface. ${ }^{[18]}$ To realize ESAs, the process is modified to dramatically (by a factor of 500) increase the thickness of the metallic patterns, allowing very low resistance, conductive antenna arms to be fabricated. ${ }^{[19,20]}$ This process can also be used to pattern metal onto extreme curvatures, such as the entire surface of a hemisphere down to its equator. The process involves stamping metallic 
(a)

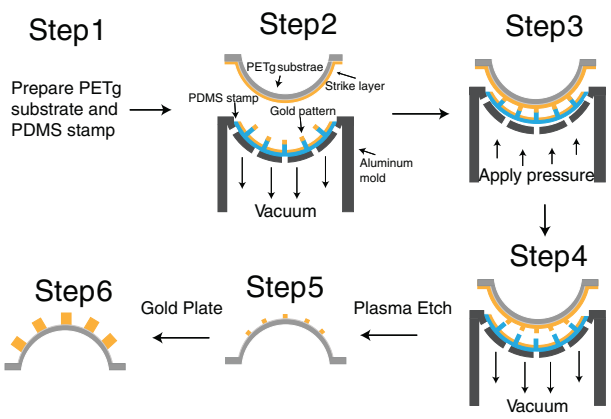

(c)

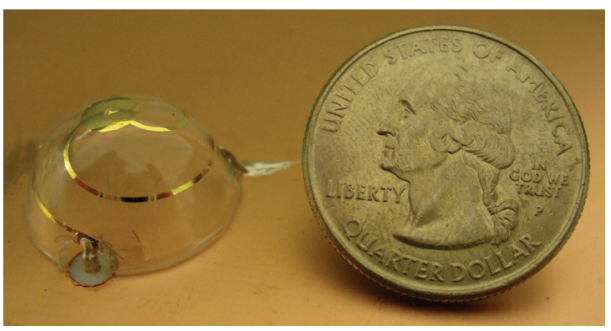

(b)

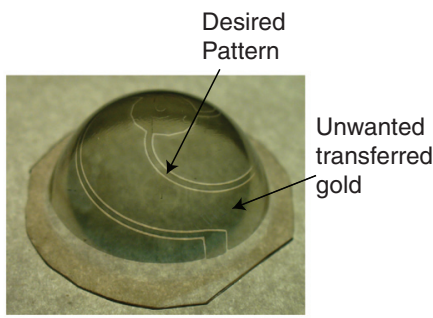

(d)

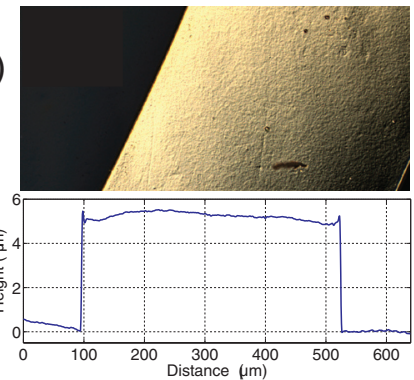

Figure 1. (a) Outline of the direct transfer patterning process that is used to stamp metallic patterns onto a contoured substrate. (b) Patterned Au on a hemispherical substrate prior to electroplating (between Step 5 and 6). Note that there are areas where unwanted Au is stamped onto the substrate, which are later removed (see experimental section). (c) The fabricated spherical helix antenna after connection to a ground plane. (d) Optical micrograph and profilometry scan of a metal conductor. The surface roughness is $0.5 \mu \mathrm{m}$.

patterns onto a contoured substrate, and then electroplating the patterns to a thickness of 5-10 $\mu \mathrm{m}$. A summary of the process is shown in Figure 1a.

The substrate used to fabricate the antenna is $0.5 \mathrm{~mm}$ thick, glycol-modified polyethylene terephthalate (PETg), which has a relative dielectric constant $\varepsilon_{r}=3.1$ and loss tangent $\tan (\delta)=$ 0.015. ${ }^{[21]}$ To begin, a flat sheet of PETg is deformed into the desired shape by heating to $150{ }^{\circ} \mathrm{C}$ (above its softening temperature), and then drawing it into a vacuum mold where it is cooled. Next, $30 \mathrm{~nm}$ thick $\mathrm{SiO}_{2}$ and $3 \mathrm{~nm}$ thick $\mathrm{Cu}$ layers are sputtered onto the contoured substrate followed by deposition of an $8 \mathrm{~nm} \mathrm{Au}$ "strike" layer. The $\mathrm{SiO}_{2}$ and $\mathrm{Cu}$ serve to improve the adhesion of $\mathrm{Au}$ to the substrate. Then, a $\mathrm{Si}$ "pattern master" is prepared by etching $40 \mu \mathrm{m}$ trenches using standard lithography and deep reactive ion etching. The location of the trenches corresponds to the final location of the metal patterns to be printed onto the substrate. A liquid polydimethyl-siloxane (PDMS) pre-polymer and hardening agent are then poured onto the Si pattern master at a 10:1 mass ratio. The PDMS is cured at $100{ }^{\circ} \mathrm{C}$ for $2 \mathrm{hrs}$, after which it is peeled from the pattern master to form a stamp. The PDMS stamp is approximately $0.5 \mathrm{~mm}$ thick, with $40 \mu \mathrm{m}$ high ridges corresponding to the pattern that is to be printed on the contoured substrate. Next, a $17 \mathrm{~nm}$ Au seed layer is added to the stamp using electron beam evaporation (Figure 1a, Step 1).

To pattern the substrate, the stamp is drawn into the same vacuum mold used to deform the substrate (Step 2). The substrate is subsequently placed in close proximity to the deformed stamp. When the vacuum is released and a small amount of pressure $(\sim 1 \mathrm{~atm})$ is applied to the back of the stamp, the ridges contact the surface of the substrate, resulting in a cold-welded metallic bond formed between the $\mathrm{Au}$ on the stamp and that on the substrate (Step 3). Further details of the cold-welding process are described in the experimental section. Then the vacuum is reapplied to separate the stamp from the substrate, transferring the $17 \mathrm{~nm}$ thick Au patterns to the surface of the contoured substrate (Step 4). Next, the substrate is etched in an Ar plasma at 30 mTorr and $150 \mathrm{~W}$ for $70 \mathrm{~s}$ to remove the strike layer that electrically connects all of the transferred patterns (Step 5). Exposure to the Ar plasma substantially heats the substrate, which is problematic since PETg has a relatively low softening temperature. To minimizing heating, the plasma is pulsed $(10 \mathrm{~s}$ on, $60 \mathrm{~s}$ off). Figure $1 \mathrm{~b}$ shows the patterned substrate after the plasma etch. Finally, the patterns are electroplated to a thickness of 5-10 $\mu \mathrm{m}$ (Step 6). Figure $1 \mathrm{~b}$ shows that there is some additional, unwanted $\mathrm{Au}$ on the substrate due to the stamping process in Step 3, which is removed using a wet Au etch following the electroplating (see experimental section). This process allows one to print arbitrary patterns onto contoured substrates, such as the spherical helix antenna shown in Figure 1c.

The minimum achievable feature size is limited by the lithography techniques used to pattern the Si pattern master, and the thickness of the electroplated conductors. Typically, feature sizes of $<10 \mu \mathrm{m}$ can be achieved using this process for $10 \mu \mathrm{m}$ thick patterns. Figure $1 \mathrm{~d}$ shows an optical micrograph and profilometry scan of an antenna's conductive arm. The surface roughness is $0.5 \mu \mathrm{m}$. We chose to use a PETg substrate because of its low cost, tolerance to the electroplating process, and vacuum forming properties. In general, any other substrate that can be shaped into the desired contour could also be utilized, but attention should be paid to its electroplating properties (see experimental section). 
Table 1. The performance of electrically small, spherical helix antennas fabricated using the direct transfer patterning process. For comparison, the performances of several alternative fabrication techniques are also provided. The direct transfer process has a slightly lower efficiency than manually bending wires, but otherwise outperforms the other fabrication techniques. Several different electrical sizes $(k a)$ are shown to demonstrate their scaling properties.

\begin{tabular}{lccccc}
\hline Fabrication Method & $\begin{array}{c}\text { Frequency } \\
(\mathrm{GHz})\end{array}$ & $k a$ & $\mathcal{Q} / Q_{l b}$ & $\eta_{\text {eff }}$ & $\begin{array}{c}\text { Half Power } \\
\text { Bandwidth }\end{array}$ \\
\hline Direct transfer patterning & 1.12 & 0.23 & 2.1 & $52 \%$ & $2.0 \%$ \\
& 1.52 & 0.31 & 1.8 & $69 \%$ & $5.3 \%$ \\
Manually bending wires & 2.70 & 0.56 & 2.2 & $88 \%$ & $14.2 \%$ \\
Silver ink printing & 0.30 & 0.38 & 1.5 & $98 \%$ & $7.6 \%$ \\
& 0.30 & 0.45 & 1.8 & $99 \%$ & $6.7 \%$ \\
Printed-circuit-board & 0.79 & 0.21 & 2.0 & $14 \%$ & $6.3 \%$ \\
& 1.70 & 0.46 & 1.5 & $71 \%$ & $15.2 \%$ \\
& 0.43 & 0.35 & 5.8 & $58 \%$ & $2.3 \%$ \\
& 1.40 & 0.50 & 5.4 & $89 \%$ & $4.1 \%$ \\
\hline
\end{tabular}

We fabricated and measured three spherical helix antennas whose performance is provided in Table 1. Figure 2 shows the dimensions of a spherical helix antenna that operates at $1.12 \mathrm{GHz}$. Each arm was designed to have 1.5 turns using Equations 1-5 in ref. [3], except that the arms begin spiraling $2 \mathrm{~mm}$ above the surface of the ground plane rather than at the ground plane. The radius of the hemisphere is $10 \mathrm{~mm}$. A $2 \mathrm{~mm}$ radius cap was placed at the top of the antenna to allow for an easy electrical connection while electroplating. Since the current near the top of the antenna is small, the cap does not affect the performance. The width of the conducting paths is $0.4 \mathrm{~mm}$, and their thickness is $5 \mu \mathrm{m}$. All antennas are impedance-matched to $50 \Omega$ with a return loss greater than $15 \mathrm{~dB}$, as

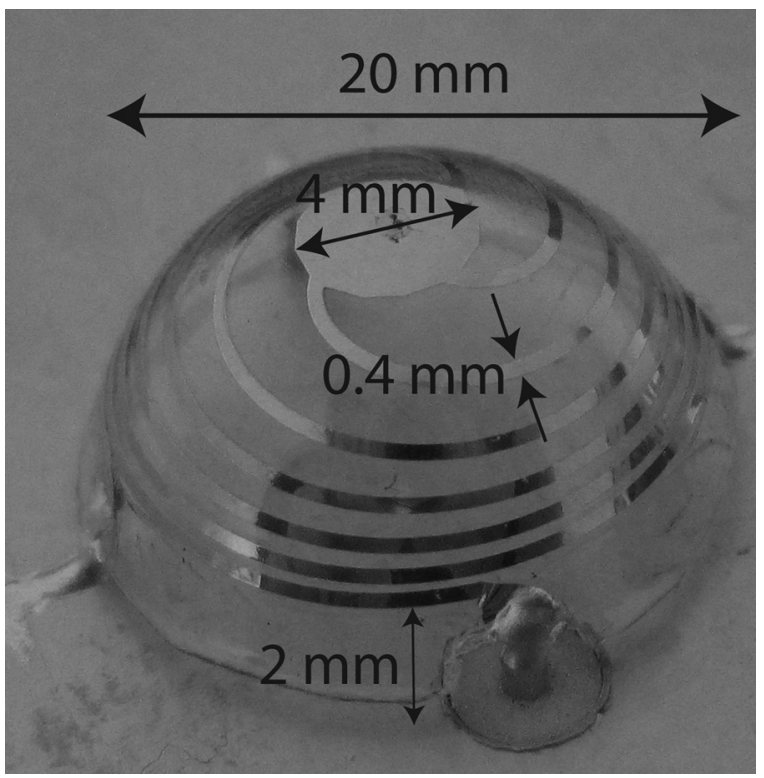

Figure 2. Dimensions of a spherical helix antenna that operates at $1.12 \mathrm{GHz}$. shown in Figure 3a. The antennas that operate at $1.52 \mathrm{GHz}$ and $2.7 \mathrm{GHz}$ are identical to that in Figure 2 except they each have 3 arms with 1.0 and 0.5 turns per arm respectively. The input impedance was used to calculate the $Q$ of the antenna. ${ }^{[17]}$ The radiation patterns of the antennas were also measured (see Figures 3b-d), and all antennas exhibit the expected electric dipole radiation patterns. Simulations also show that as the size of the ground plane increases, the dipole radiation patterns approach those of a monopole, as expected.

Using the stamping process, three different spherical helix antennas were designed and fabricated to operate at three different frequencies. These results allow for the direct comparison of the antennas with previously published data of similar electrical sizes. To highlight the effect of the fabrication method on antenna performance, Table 1 summarizes the results of some competing fabrication methods such as manually bending wires, ${ }^{[3]}$ silver ink printing, ${ }^{[1]}$ and conventional 2D printed circuit board (PCB) techniques. ${ }^{[6]}$

Manually fabricated antennas provide the best performance since they have a very low $Q$ and high efficiency. The increased $Q$ of the direct transfer patterning technique primarily results from the presence of a substrate. ${ }^{[9]}$ The reasons for the decreased efficiency of the antenna fabricated using direct transfer patterning is the additional substrate loss, and the lower skin depth due to the higher frequency of operation. ${ }^{[22]}$ Although manual fabrication offers improved performance, manually bending wires is a time consuming and potentially expensive process. Silver ink printing achieves comparable $Q$ 's, but significantly lower efficiency than direct transfer patterned devices since the conductivity of the ink is only $30 \%$ that of copper. ${ }^{[1]}$ This lower efficiency leads to an increased bandwidth of the silver ink printed antennas. In addition, the ink printing process is relatively slow since every conductive trace needs to be individually drawn. Finally, conventional two-dimensional printed circuit board fabrication offers a significantly higher $Q$ and lower efficiency since the antennas do not take advantage of the volume provided by a spherical geometry.

In summary, we present a process to rapidly stamp antennas onto arbitrarily contoured substrates. We demonstrate the ability to fabricate antennas whose bandwidths approach the maximum achievable limit for a given electrical size. It is shown that direct transfer patterning can produce electrically small antennas that are significantly more efficient than previously published methods and potentially less expensive to fabricate. In addition, this technology allows components with small features, (e.g., interdigitated capacitors or densely packed metallic conductors) to be placed onto arbitrarily contoured substrates. This has potential applications in diverse areas such as high gain antennas, conformal antenna arrays, RFIDs, metamaterials, and transformation optics. ${ }^{[14,16,23]}$

\section{Experimental Section}

Cold-welding: The direct transfer patterning process relies on forming a cold-welded bond between the stamp and substrate (Figure 1a, Step 3). For cold-welding to occur, the two surfaces must be coated with identical metal layers and brought into intimate contact so that they adhere. ${ }^{[24-27]}$ The surfaces must be clean and smooth since small dust particles or surface nonplanarity will inhibit intimate contact. Elastomers such as 

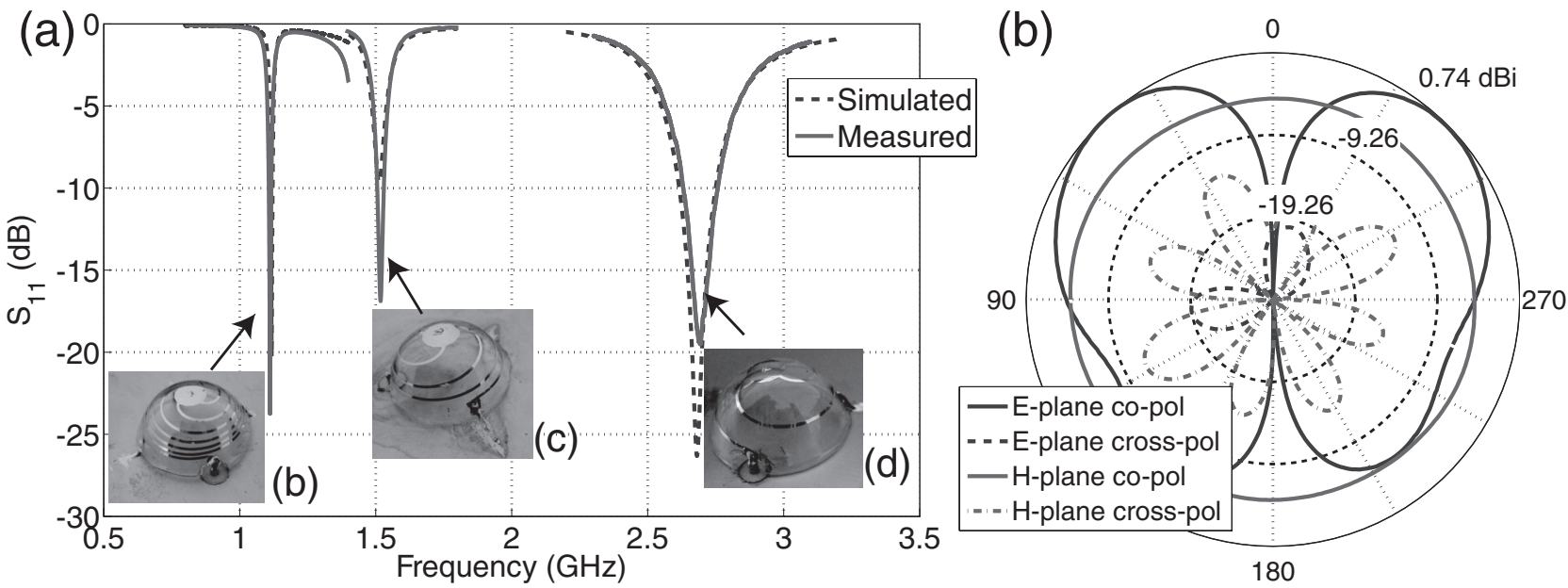

(c)

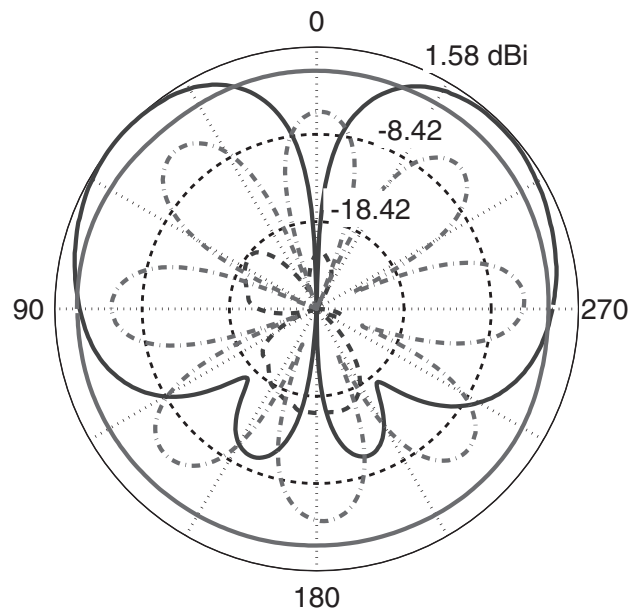

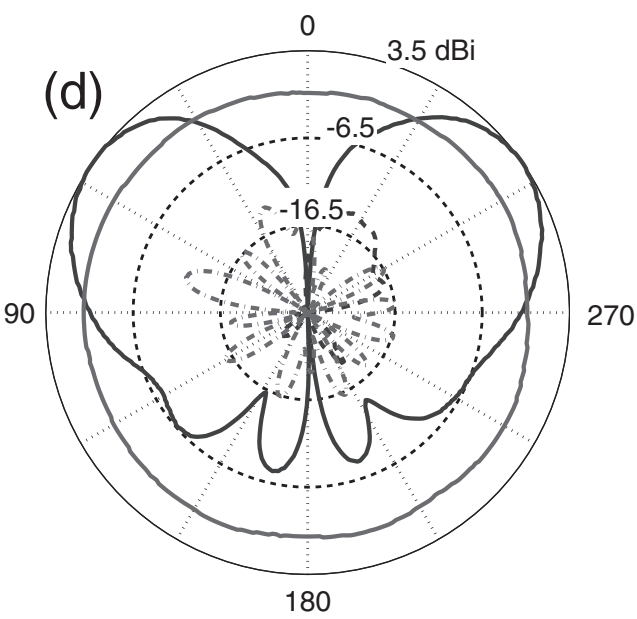

Figure 3. (a) Reflection coefficient of the antennas compared with simulation. (b)-(d) Co-polarization and cross-polarization gain patterns along the electric $(E)$ and magnetic $(\mathrm{H})$ field planes of the spherical helix antennas operating at $1.12 \mathrm{GHz}, 1.52 \mathrm{GHz}$, and $2.7 \mathrm{GHz}$, respectively.

PDMS can mold themselves around surface defects, thereby limiting their adverse effects. ${ }^{[24,26]}$ In direct transfer patterning, it is desirable to transfer metal thicknesses that are significantly thicker than the strike layer to provide a high contrast for the plasma etch (Figure 1a, Step 5). It has been shown that thicknesses up to $20 \mathrm{~nm}$ can be transferred using this process. ${ }^{[18]}$ However, greater thicknesses were difficult to obtain, possibly due to wrinkling of the PDMS during the metal deposition. ${ }^{178,28]}$

Electroplating: To maximize antenna efficiency, the conductor thickness should be at least $3 \delta_{\mathrm{s}}$, where $\delta_{\mathrm{s}}$ is the skin depth $(\sim 2 \mu \mathrm{m}$ at L-band) at the operating frequency. Several factors influence the optimal metal plating thickness. When the metal is plated too thick, it becomes overly stressed, forcing it to "peel" off the substrate. Rougher substrates can employ thicker metal films due to increased mechanical anchoring. ${ }^{[29]}$ The surface roughness of the PETg substrate is $14 \mathrm{~nm}$, measured using atomic force microscopy (AFM). For $\mathrm{Cu}$ and $\mathrm{Au}$ plating, we achieved a thickness of 12 and $7 \mu \mathrm{m}$, respectively, without the metal peeling. In addition, the use of adhesion layers can significantly increase the maximum thickness of the metal. We found that $\mathrm{SiO}_{2}$ and $\mathrm{Cu}$ adhesion layers work well because they offer sufficient adhesion to electroplate to the desired thickness, while $\mathrm{Cu}$ is easy to remove during the Ar plasma etch. ${ }^{[30]}$

Removing interstitial Au: It can be seen in Figure $1 \mathrm{~b}$ that there is additional $\mathrm{Au}$, between the helical conductors. When pressure is applied to the back of the stamp, its ridges contact the substrate to form a coldwelded metallic bond. When these ridges are spaced sufficiently far apart, additional areas of the flexible PDMS stamp also contact the substrate, thereby creating transfer of $\mathrm{Au}$ in between the conductive traces. The interstitial Au can be removed using a wet Au etch (Transene TFA Au Etch). Following the plasma etch, there is a gap between the unwanted $\mathrm{Au}$ and the desired pattern. This gap creates electrical isolation such that only the desired pattern is electroplated. After electroplating, a wet Au etch is used to remove the $17 \mathrm{~nm}$ thick, unwanted Au with minimal effect on the electroplated pattern.

Antenna measurements: The antennas were printed onto a $10 \mathrm{~mm}$ radius PETg hemisphere. All but one of the arms was connected to a $200 \mathrm{~mm} \times 200 \mathrm{~mm}$ ground plane using Ag-loaded epoxy, while the remaining arm was connected to the inner conductor of a coaxial transmission line acting as an antenna feed. The input impedance of the antenna was measured using a vector network analyzer (Agilent E8361A).

The radiation efficiency of the antennas was measured using both the Wheeler cap method and the gain comparison method. ${ }^{[10,31]}$ In the Wheeler cap method, the input impedance of the antenna is measured in free space. Next, a Wheeler cap is placed over the antenna and secured to the ground plane, and the input impedance is measured again. These two measurements are used to find the radiation and loss resistances, and therefore the radiation efficiency. The accuracy of this method can be 
improved when postprocessing the data by adding an ideal transmission line to rotate the input impedance around the Smith chart, such that it resembles a constant resistance or conductance circle. ${ }^{[32]}$

The radiation patterns were measured using the University of Michigan's $18 \mathrm{~m}$ anechoic chamber. The antenna under test (AUT) is placed on a rotating styrofoam platform in the quiet zone of the chamber. The AUT is then connected to a signal generator (Agilent N5183A). At the other end of the chamber is a receiving, standard gain horn antenna connected to a spectrum analyzer (Hewlett Packard 8592L) to measure the received power. By rotating the AUT and measuring the power received by the horn antenna, the radiation patterns are measured. Then the radiation efficiency is found using the gain comparison method. ${ }^{[31]}$ For this, the AUT is replaced by a reference horn antenna with a known gain. By comparing the received power of the AUT to the reference, the gain and radiation efficiency are determined. The radiation efficiency measured using the gain comparison method agreed to within $2 \%$ of the efficiency found using the Wheeler cap method.

Finally, the radiation quality factor (Q) was found using the measured input impedance of the antenna. It can be shown that for single resonant antennas, the $Q$ is related to the input impedance through the following relation, ${ }^{[17]}$

$Q\left(\omega_{0}\right)=\frac{\omega_{0}}{2 R_{0}}\left|\frac{d Z\left(\omega_{0}\right)}{d \omega}\right|$

Here, $\omega_{0}$ is the resonant frequency of the antenna, $R_{0}$ is the input resistance, and $\frac{d Z\left(\omega_{0}\right)}{d \omega}$ is the frequency derivative of the input impedance evaluated at the resonant frequency.

\section{Acknowledgements}

The authors would like to thank Pilar Herrera-Fierro for her assistance in fabrication. The fabrication was performed using the University of Michigan, Lurie Nanofabrication Facility. This work was supported by a Presidential Early Career Award for Scientists and Engineers (FA955009-1-0696), a NSF Faculty Early Career Development Award (ECCS0747623), and Universal Display Corp.

Received: November 9, 2011

Revised: December 6, 2011

Published online: January 30, 2012

[1] J. J. Adams, E. B. Duoss, T. F. Malkowski, M. J. Motala, B. Y. Ahn, R. G. Nuzzo, J. T. Bernhard, J. A. Lewis, Adv. Mater. 2011, 23, 1335-1340.

[2] S. R. Best, IEEE Trans. Antennas Prop. 2003, 51, 1292-1300.

[3] S. R. Best, IEEE Trans. Antennas Prop. 2004, 52, 953-960.
[4] S. R. Best, D. L. Hanna, IEEE Antennas Prop. Mag. 2010, 52, 47-70.

[5] L. J. Chu, J. Appl. Phys. 1948, 19, 1163-1175.

[6] A. Erentok, R. W. Ziolkowski, IEEE Trans. Antennas Prop. 2008, 56, 691-707.

[7] J. S. McLean, IEEE Trans. Antennas Prop. 1996, 44, 672-676.

[8] H. R. Stuart, C. Tran, IEEE Antennas Wireless Prop. Lett. 2007, 6, 7-10.

[9] H. Thal, IEEE Trans. Antennas Prop. 2006, 54, 2757-2763.

[10] H. A. Wheeler, Proc. IRE 1959, 47, 1325-1331.

[11] A. D. Yaghjian, H. R. Stuart, IEEE Trans. Antennas Prop. 2010, 58, 3114-3121.

[12] O. S. Kim, IEEE Trans. Antennas Prop. 2010, 58, 2210-2217.

[13] R. Bhattacharya, S. Wagner, Y. J. Tung, J. R. Esler, M. Hack, Proc. IEEE 2005, 93, 1273-1280.

[14] D. Y. Shin, Y. Lee, C. H. Kim, Thin Solid Films 2009, 517, 6112-6118.

[15] B. Tomasic, J. Turtle, S. Liu, R. Schmier, S. Bharj, P. Oleski, IEEE Int. Symp. Phased Array Sys. Tech. 2003, 411-416.

[16] J. Pendry, D. Schurig, D. Smith, Science 2006, 312, 1780.

[17] A. D. Yaghjian, S. R. Best, IEEE Trans. Antennas Prop. 2005, 53, 1298-1324.

[18] X. Xu, M. Davanco, X. Qi, S. R. Forrest, Org. Electron. 2008, 9, $1122-1127$.

[19] C. Pfeiffer, X. Xu, S. R. Forrest, A. Grbic, Int. Symp. on Antenna Tech. and Applied Electromagnetics (ANTEM), Ottawa, ON2010.

[20] C. Pfeiffer, X. Xu, S. R. Forrest, A. Grbic, IEEE Int. Symp. on Antennas and Prop., Spokane, WA2011.

[21] R. Pelster, IEEE Trans. Microwave Theory Techniques 1995, 43, 1494-1501.

[22] S. R. Best, A. D. Yaghjian, IEEE Antennas Wireless Prop. Lett. 2004, 3, 314-316.

[23] B. Tomasic, J. Turtle, S. Liu, Int. Union of Radio Science, XXVIIth General Assembly 2002, 3161-3164

[24] G. S. Ferguson, M. K. Chaudhury, G. B. Sigal, G. M. Whitesides, Science 1991, 253, 776-777.

[25] C. Kim, P. E. Burrows, S. R. Forrest, Science 2000, 288, 831.

[26] C. Kim, S. R. Forrest, Adv. Mater. 2003, 15, 541-545.

[27] C. Kim, M. Shtein, S. R. Forrest, Appl. Phys. Lett. 2002, 80, 4051.

[28] N. Bowden, S. Brittain, A. G. Evans, J. W. Hutchinson, G. M. Whitesides, Nature 1998, 393, 146-149.

[29] D. De Leeuw, P. Kraakman, P. Bongaerts, C. Mutsaers, D. Klaassen, Synt. Met. 1994, 66, 263-273.

[30] M. Seah, Thin Solid Films 1981, 81, 279-287.

[31] C. A. Balanis, Antenna Theory: Analysis and Design, 3rd ed., John Wiley \& Sons, New York, NY 1997.

[32] W. McKinzie III, IEEE Int. Symp. on Antennas and Prop., Montreal, Que., Canada 1997, 542-545. 